\title{
gु \\ Force-controlled lifting of molecular wires
}

\author{
N. Fournier, C. Wagner, C. Weiss, R. Temirov, ${ }^{*}$ and F. S. Tautz \\ Peter Grünberg Institut (PGI-3), Forschungszentrum Jülich, 52425 Jülich, Germany and \\ JARA-Fundamentals of Future Information Technology, 52425 Jülich, Germany
}

(Received 12 May 2011; published 25 July 2011)

\begin{abstract}
Lifting a single molecular wire off the surface with a combined frequency-modulated atomic force and tunneling microscope it is possible to monitor the evolution of both the wire configuration and the contacts simultaneously with the transport conductance experiment. In particular, critical points where individual bonds to the surface are broken and instabilities where the wire is prone to change its contact configuration can be identified in the force gradient and dissipation responses of the junction. This additional mechanical information can be used to unambiguously determine the conductance of a true molecular wire, that is, of a molecule that is contacted via a pointlike "crocodile clip" to each of the electrodes but is otherwise free.
\end{abstract}

DOI: 10.1103/PhysRevB.84.035435

PACS number(s): 82.37.Gk, 73.63.-b, 81.07.-b

Recently it has been demonstrated that the application of scanning tunneling microscopy (STM) to single-molecule transport studies increases the degree of experimental control considerably, because the conformation and the local environment of the molecule before and after the transport measurement can be determined. ${ }^{1-6}$ In those instances when the molecule can be contacted in a point-contact-like manner, essentially like an atom, this degree of control is sufficient to make transport measurements fully reproducible; examples are conductance measurements of $\mathrm{C}_{60} \cdot{ }^{1,3,5,6}$ In the more general cases of ribbonlike or platelet molecules, however, the molecule must be manipulated into a free-standing configuration in which it remains connected to the junction electrodes (i.e., substrate surface and STM tip) via the two pointlike contacts. As no direct methods exist to control and monitor the molecule during this manipulation, its configuration in the junction is typically deduced from the transport data itself or from theoretical simulations. ${ }^{7-9}$ This approach, however, often leads to ambiguities with respect to the molecular conformation and thus cannot yield detailed and reliable characterizations of molecular junctions.

In this work we propose a new approach to single-molecule transport measurements based on, first, contacting a surfaceadsorbed molecule with the tip of a combined atomic force and tunneling microscope (AFM/STM), second, lifting up the molecule into the free-standing wire configuration on a trajectory that has been optimized before in a simulation, and third, verifying the proper execution of the lifting by monitoring online the AFM signal that measures the junction's stiffness. We show that molecular wire junctions formed in this way can be characterized reproducibly. For our proofof-principle experiment we have chosen the well-studied system of 3,4,9,10-perylene-tetracarboxylicacid-dianhydride (PTCDA). ${ }^{10}$ The structure of PTCDA, shown in Fig. 1, can be understood as a graphene nanoribbon ${ }^{11}$ with functional groups attached at both ends. Therefore, PTCDA represents a wider class of molecular wires which consist of two separated parts: first, a conducting $\pi$-conjugated core and, second, clamps at its ends which provide a mechanically stable electronic coupling of the wire's conducting body to the electrodes. ${ }^{2,12}$

For our experiments we prepared an atomically clean $\mathrm{Ag}(111)$ surface in UHV by successive cycles of $\mathrm{Ar}^{+}$ sputtering and annealing at $550{ }^{\circ} \mathrm{C}$. A submonolayer coverage of PTCDA molecules was evaporated onto the $\mathrm{Ag}$ (111) surface kept at RT from a home-built Knudsen cell at a temperature of $300{ }^{\circ} \mathrm{C}$. Finally, isolated PTCDA molecules on $\operatorname{Ag}(111)$ were produced by detaching them with the STM tip from the edge of a PTCDA island. ${ }^{2}$

In detail, we contact the single, isolated PTCDA molecule, which initially is adsorbed flat on a single-crystal $\operatorname{Ag}(111)$ surface, with the atomically sharp Ag-covered tungsten tip of a low temperature CREATEC STM/AFM (based on the qPlus tuning fork design ${ }^{13}$ ). Moving the tip into contact with the molecule and then retracting it away from the surface, we bring the molecule into the free-standing wire geometry, in which the molecule is bound to the surface on one side and to the tip on the other, each via a carboxylic oxygen atom (Fig. 1). To gain full control over the lifting process, we retract the tip along a trajectory that minimizes lateral forces in the junction. Lateral forces can lead to abrupt sliding of the molecule on the surface and undermine external control of the junction structure. ${ }^{2,14}$ The trajectory with vanishing lateral forces is shown in Fig. $1^{14}$ and was obtained from force-field simulations that take into account both the chemical bonding and the van der Waals interaction between the molecule and the electrodes.

Force-field calculations have been carried out for the tip/PTCDA/Ag(111) junction. The tip was modeled by a single $\mathrm{Ag}$ atom. The $\mathrm{Ag}(111)$ surface was modeled by one atomic layer. The interaction between the atoms within the PTCDA molecule was described by the AMBER ${ }^{15}$ parameter set. The tip-molecule interaction is modeled by a Morse potential (depth $1 \mathrm{eV}$ ) acting between the tip atom and one of the carboxylic oxygens of PTCDA. ${ }^{12}$ The molecule-substrate interaction is modeled by interaction potentials between individual atoms constituting the molecule and the surface as a whole. The interaction potential for each species in the molecule $(\mathrm{C}$, $\mathrm{H}$, carboxylic $\mathrm{O}$, anhydride $\mathrm{O}$ ) with $\mathrm{Ag}$ is represented by a one-dimensional $z$-dependent Morse potential; for each of these potentials, the position of the energy minimum and the depth has been chosen separately to reproduce the available experimental data, namely the adsorption height of PTCDA on $\operatorname{Ag}(111),{ }^{16-18}$ its deformation, ${ }^{16-18}$ and the adsorption energy for PTCDA/Au(111), ${ }^{19}$ which is taken as a lower boundary 


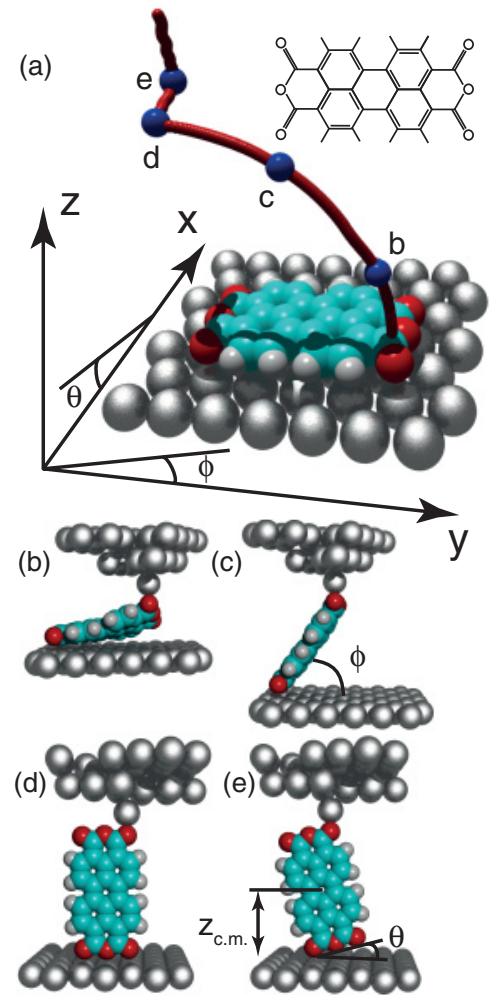

FIG. 1. (Color) Simulated junction geometries during tip retraction. (a) PTCDA adsorbed on $\operatorname{Ag}(111)$. The molecule is shown in the adsorption site that is used in the simulation. The tip trajectory employed in experiment and simulation is shown in red (its projection into the $x y$ plane is shown as a shadow). An animation of this tip trajectory is provided in the supplemental material (Ref. 14). Tip positions corresponding to the four junction geometries in panels (b) to (e) are marked in blue. The two angles $\phi$ and $\theta$ describe the orientation of the molecule in the junction. (b) The second carboxylic oxygen is detached from the surface. (c) The almost planar molecule is lifted into the upright configuration. (d) Upright molecule $\left(\phi=90^{\circ}, \theta=0^{\circ}\right)$ bound to $\operatorname{Ag}(111)$ via two carboxylic oxygen atoms. (e) Molecular wire configuration, that is, $\phi=90^{\circ}, \theta=15^{\circ}$. The coordinate $z_{c . m}$. describes the motion of the molecule after it is detached from the surface.

for the adsorption energy for PTCDA/Ag(111). Finally, the potentials have been fine-tuned to optimize the fit to the experimental $\frac{d F}{d z}(z)$ data. The corrugation of the interaction potential between the carboxylic $\mathrm{O}$ atoms and the surface has been set to $40 \mathrm{meV}$, according to the activation energy of PTCDA diffusion that was measured on $\operatorname{Ag}(100) .{ }^{20}$ The force-field simulations have been carried out for a PTCDA molecule that is aligned along the high symmetry direction of $\operatorname{Ag}(111) .^{21}$ In the simulations the tip is retracted in steps of $0.25 \mathrm{pm}$. After each step the junction geometry is relaxed. To define the tip trajectory of Fig. 1(a), the lateral position of the tip apex atom is adjusted at each tip height $z_{\text {tip }}$ such that all lateral forces on the tip vanish. The thus-obtained tip trajectory has also been used in the experiments.

To measure the junction stiffness experimentally during tip retraction, we record the frequency shift $\Delta f$ of the tuning fork oscillations. In our experiments we have used qPlus sensors from Createc Fischer GmbH. They consist of a tungsten tip that is glued to the quartz tuning fork. The tip was etched electrochemically. Then it was prepared in situ by crashing into the clean $\operatorname{Ag}(111)$ surface at a bias voltage of $V=100 \mathrm{~V}$ with the current limited to $1 \mathrm{~mA}$. The qPlus sensor $\left(k_{0}=1800 \mathrm{~N} / \mathrm{m}\right)$ oscillates with its resonance frequency $f_{0} \approx 20.9 \mathrm{kHz}$ and an amplitude of $A_{0} \approx 0.1-0.2 \AA$. The $\Delta f$ signal can be directly related to the stiffness (or force gradient) $k=\frac{d F}{d z} \approx-\frac{2 k_{0}}{f_{0}} \Delta f$ of the molecular wire junction, where $F$ is the force acting on the apex of the tip..$^{22,23}$ The inset of Fig. 2(a) displays $\Delta f$ data of a single cycle of tip approach toward the not-yetcontacted molecule (black) and tip retraction after contacting the molecule (red). The black curve shows a decreasing stiffness due to the increasing attraction between the tip and the substrate (the latter consisting of both the PTCDA

(a)

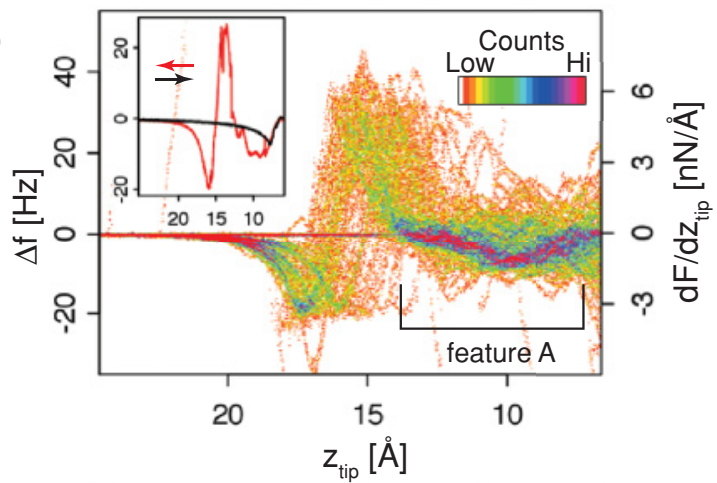

(b)

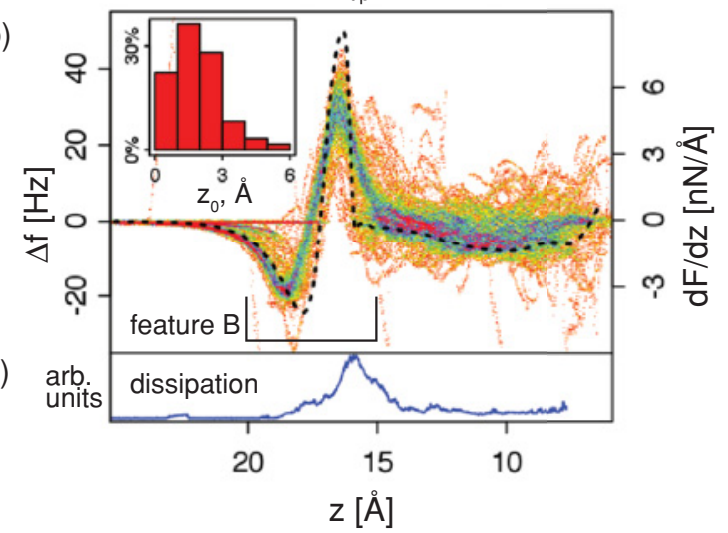

FIG. 2. (Color) Stiffness of the tip/PTCDA/Ag(111) junction. (a) Two-dimensional histogram of $121 \Delta f(z)$ curves recorded while lifting up single isolated PTCDA molecules. After each manipulation cycle the molecule remained at the tip. In $65 \%$ of the cases it was possible to redeposit the molecule back onto the surface by having the tip approach the surface and applying a voltage of $+0.6 \mathrm{~V}$ to the sample. The inset shows $\Delta f(z)$ for a single approach/retraction cycle: Approach is shown in black, retraction in red. For all curves in the histogram, the $\Delta f$ signal during tip approach was subtracted from the $\Delta f$ data taken during retraction. (b) Same dataset as in panel (a), but each curve is shifted on the horizontal axis by individual values $z_{0}$ such that the peak belonging to feature $\mathrm{B}$ is aligned with the corresponding peak in the simulated $\Delta f$ curve (dashed line) (Ref. 24). The inset shows a histogram of shift distances $z_{0}$. (c) Averaged dissipation signal for all 121 retractions of panel (b). Before averaging, each curve was shifted by the same $z_{0}$ as the corresponding $\Delta f$ curve in panel (b). The dissipation is a measure of the energy needed to sustain a constant oscillation amplitude of the tuning fork sensor. 
molecule and the metal surface). At the end of the approach, the jump-into-contact of the PTCDA molecule to the tip apex can be discerned as a sharp kink in the stiffness curve. To exclude long-range forces that are not related to the lifting of the molecule from our analysis, we subtract the approach curves (background) from the retraction curves. The resulting background-subtracted retraction curves of all 121 approach cycles that we have performed are shown as a two-dimensional color-coded histogram in Fig. 2(a).

In the range $z_{\text {tip }}=5$ to $14 \AA{ }^{25}$ Fig. 2(a) exhibits a shallow dip in the junction stiffness $\frac{d F}{d z \text { tip }}$, which we label as feature A. When the tip is retracted beyond $z_{\text {tip }} \approx 14 \AA$, the stiffness curves in Fig. 2(a) scatter more strongly. Nevertheless, it is evident that most of the curves show a characteristic peak/dip structure, to which we refer as feature B from now on. In Fig. 2(b), we have aligned all individual stiffness curves at feature B, with the result that in the range $z=16$ to $24 \AA$, the experimental curves collapse onto one. Apparently, the macroscopically measured $z_{\text {tip }}$ does not characterize the configuration of the molecule in the junction unambiguously. This ambiguity may, for example, arise due to different bonding positions of the carboxylic oxygen atom of PTCDA on the tip. In the remainder of the paper, we exclusively discuss the aligned stiffness curves of Fig. 2(b), because feature B defines a reference point for the microscopic $z$ coordinate, as will become clear below. This coordinate $z$ is related to $z_{\text {tip }}$ by $z=z_{\text {tip }}+z_{0}$, where $z_{0}$ is an offset that varies from experiment to experiment [inset, Fig. 2(b)].

Before discussing Fig. 2 in detail, it is necessary to analyze which part of the junction the measured stiffness signal $\frac{d F}{d z}$ is related to. Because we have never observed structural changes of either the tip or the $\operatorname{Ag}(111)$ surface in our experiments, we conclude that the $\operatorname{Ag}(111)$ surface and the tip apex are so stiff that their small deformations occur within the harmonic limit $\left(\frac{d F}{d z}=\right.$ const). Consequently, any influence of these deformations on the curves in Fig. 2 can be ruled out. The three remaining elements which could in principle affect the $\frac{d F}{d z}$ curves in Fig. 2 are the molecule itself and its bonds to the surface and to the tip. According to ab initio calculations ${ }^{12}$ and our experimental data, which show that at the end of the approach-retraction cycle the PTCDA molecule nearly always remains connected to the tip, we conclude that the tip-molecule bond is the stiffest. Hence, it should deform much less than the molecule and its bond to the surface. We can therefore conclude that the measured $\frac{d F}{d z}$ curves are a property of the PTCDA molecule itself and of the PTCDA/Ag(111) surface contact, revealing their response to the chosen tip trajectory. This illustrates the benefits of using stiff sensors for force spectroscopy: If the sensor is much stiffer than the probed object, its properties do not affect the measurement. ${ }^{26}$ In particular, unlike traditional force spectroscopy techniques, which work only until bond ruptures occur, ${ }^{27,28}$ stiff sensors like the qPlus offer the possibility to coerce the molecular junction smoothly through bond rupture processes. This is vital in the present context, because it allows the stabilization of the molecular junction in any desired configuration along the chosen trajectory.

With this knowledge, we are able to interpret the experimental stiffness curves $\frac{d F}{d z}(z)$ in Fig. 2(b) by comparing them

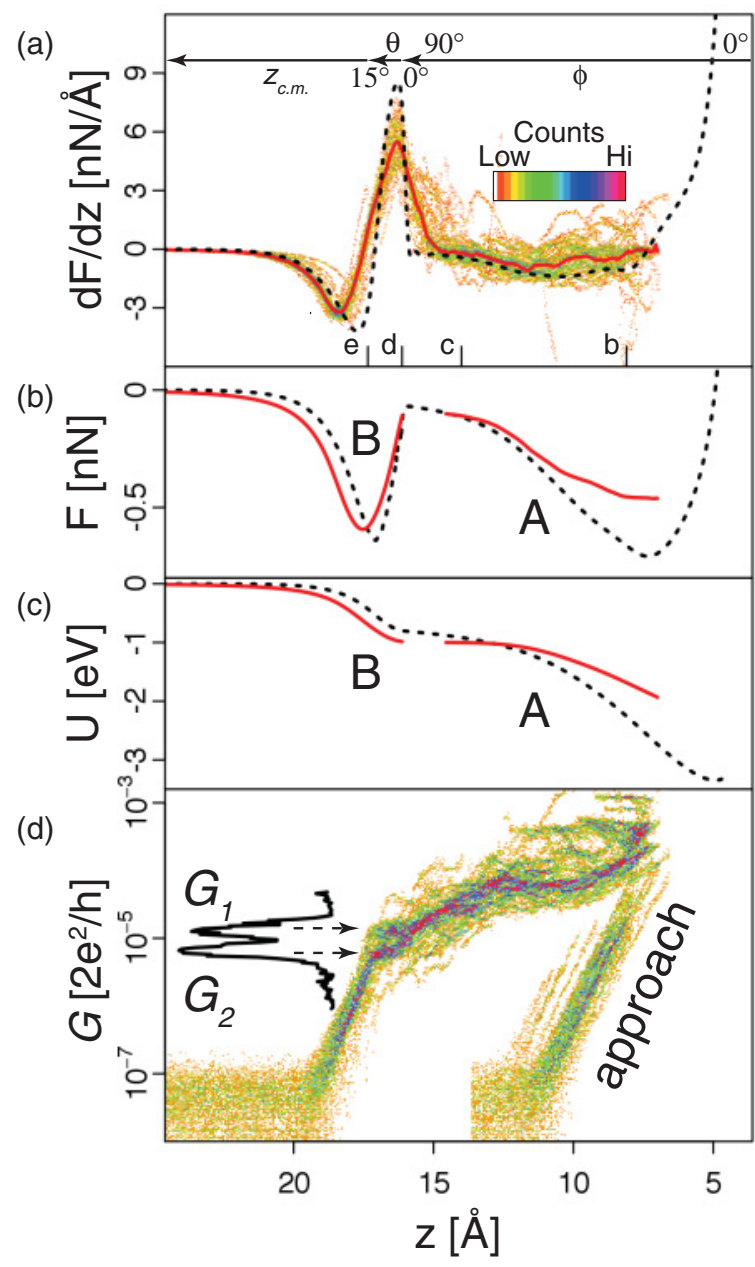

FIG. 3. (Color) Force, potential energy, and conductance of the molecular wire. (a) Histogram of the 35 integrable $\frac{d F}{d z}$ curves (i.e., no abrupt jumps for $z>15 \AA$ ) from the dataset in Fig. 2(b). The solid red line displays the averaged experimental data. The black dashed line shows the simulated $\Delta f$ curve (Ref. 24). The tip heights that correspond to the junction geometries in Fig. 1 are indicated and labeled as b, c, d, and e. The upper horizontal axis $\left(\phi, \theta, z_{c . m}\right)$ indicates the coordinates of molecular motion, enforced by the tip trajectory shown in Fig. 1(a). (b) Black dashed line, force on the tip as calculated in the force-field simulation; red solid lines, force on the tip as calculated by integrating the averaged experimental $\frac{d F}{d z}$ in panel (a) [red solid line in panel (a)] for $z>16 \AA$ and for $z<15 \AA$. The branch for $z<15 \AA$ was shifted along the vertical axis such that it starts at the value where the left section of the curve ended. (c) Black dashed line, potential energy of PTCDA in the junction as calculated in the force-field simulation; red solid lines, potential energy of PTCDA in the junction as calculated by integrating the experimental $F(z)$ curves in panel (b) [red solid line in panel (b)]. The integration constant for $z<15 \AA$ was again chosen to match the value where the left section of the curve ended. (d) Histogram of the junction conductance $\frac{d I}{d V}(z)$ at a bias voltage of $V=-0.5 \mathrm{mV}$ for the same dataset as in panels (a)-(c). At $z \approx 17 \AA$ the last molecule-substrate bond breaks and the junction enters the tunneling regime. The inset is a line profile through the histogram parallel to the vertical axis in the corridor between $z=16.25 \AA$ and $z=17.0 \AA$.

to a simulated $\frac{d F}{d z}(z)$ curve [black dashed lines in Figs. 2(b) and 3(a)], which is obtained from the same force-field model 
that was used to extract the tip retraction trajectory. ${ }^{24}$ For this comparison, we remove all curves from the experimental dataset in which the lifting of the molecule ends prematurely, for example, because the molecule instantaneously snaps to the tip. The remaining histogram in Fig. 3(a) is in very good agreement with the simulation, particularly for $z>16 \AA$, indicating that the lifting process in the corresponding experiments proceeds as predicted by the simulation. This conclusion is confirmed by the more detailed analysis in the next paragraph.

Because it is more intuitive to discuss the junction in terms of its total energy $U(z)$ and the force $F(z)=\frac{d U}{d z}$ than in terms of its stiffness $\frac{d F}{d z}=\frac{d^{2} U}{d z^{2}}$, we turn to the simulated $F(z)$ [dashed line in Fig. 3(b)] and $U(z)$ [dashed line in Fig. 3(c)] with the aim to understand the origin of features $A$ and $B$ in the measured stiffness curves. The simulated $F(z)$ and $U(z)$ reveal that feature $\mathrm{A}$ is associated with the molecule being lifted out of a shallow attractive potential as the tip moves away from the surface. Considering the corresponding configurations in Fig. 1, it becomes clear that this attractive potential $\mathrm{A}$ is due to the delocalized $\pi$ bond between PTCDA and $\operatorname{Ag}(111)$, which is broken gradually as the tip is retracted from 7 to $15 \AA$. According to Fig. 1 and animation 2 in the supplemental material, ${ }^{14}$ in this range the retracting tip essentially changes the angle $\phi$ between the molecule and the surface plane. At $z \approx 16 \AA$ (point d), the tip trajectory changes [cf. Fig. 1(a)], and the motion of the molecule is from there on constrained to a different coordinate when the tip is retracted further. From Fig. 1 and animation 2 in the supplemental material ${ }^{14}$ it is clear that the relevant new coordinates are the angle $\theta$ until point $\mathrm{e}(z \approx 17 \AA)$, and the $z$ position of the center of mass of the PTCDA molecule, $z_{\text {c.m. }}$, beyond point e $(z>$ $17 \AA$ ). The simulated $F(z)$ and $U(z)$ in Figs. 3(b) and 3(c) show that immediately after the coordinate change $\phi \rightarrow\left(\theta, z_{c . m .}\right)$ the molecule is again lifted out of an attractive potential (potential B). Therefore, we can conclude that feature B in the experimental stiffness curves originates from the interaction of the lower end of the vertically upright molecule with the surface. Outside this potential well the molecule is completely removed from the surface and both $F$ and $U$ approach zero (at $z \approx 24 \AA$ ).

Summarizing up to this point, we have seen that the shape of the experimental stiffness curves verifies that the target trajectory is executed in the experiments as predicted by the simulation. In other words, starting from the surface-adsorbed molecule that has been contacted with the STM tip, a freestanding molecular wire of controlled geometry is created in a systematic and reproducible manner. Unlike in the simulation, however, the real junction passes through an instability at $z \approx 16 \AA$, as evidenced by the sharp peak in the dissipation signal of Fig. 2(c). In dynamic AFM, energy dissipation arises whenever the work $\int F d s$ in the downward and upward half cycles of the tip oscillation is not the same. In the present case, this indicates that the configuration of the molecule in the junction changes within one cycle. We have seen in the previous paragraph that at $z \approx 16 \AA$, where the maximum in the dissipation signal appears, the molecule stands upright in the junction and the change in coordinate $\phi \rightarrow \theta$ takes place. It is clear that in this configuration the oscillations of the tip (which, incidentally, are not contained in the simulation) may lead to particularly large compressive or tensile stress in the junction. This, in turn, makes the molecule prone to change its configuration in the junction. The presence of the sharp dissipation maximum at $z \approx 16 \AA$ therefore confirms that at this distance the molecule in the junction stands indeed upright, and thereby it also confirms our interpretation of features $A$ and $\mathrm{B}$ to either side of the dissipation maximum.

To compare experiment and simulation quantitatively, we have integrated the averaged experimental stiffness of Fig. 3(a) (red line), once to yield the force $F$ [Fig. 3(b), red line] and twice to yield the potential energy $U$ [Fig. 3(c), red line]. It is clear that this integration cannot be extended meaningfully across the dissipative region because here the molecule traverses different trajectories with different forces in the upward and downward half cycles of the tip oscillation, and hence the measured frequency shift $\Delta f$ is not any more related to a unique stiffness in a well-defined configuration. The sharp dissipation maximum at the instability therefore divides the lifting process into two regimes, both of which must be integrated separately. Starting from $z=24 \AA$, where the force and the potential energy can be set to zero to fix the integration constants, we have integrated the average experimental curve of Fig. 3(a) up to $z=16 \AA$. The results for both $F$ and $U$ agree very well with their simulated counterparts [cf. dashed lines in Figs. 3(b) and 3(c)]. We can therefore conclude that the maximal force that has to be overcome to remove the upright molecule from the surface is $0.6 \mathrm{nN}$. This force corresponds to a binding energy in this configuration of $1 \mathrm{eV}$. This binding energy includes contributions both from the short-range (chemical) interaction between the carboxylic oxygen atoms and the surface as well as long-range van der Waals interaction between the whole of the molecule and the surface. For the integration from $z=5 \AA$ to $z=14 \AA$, there is no experimentally accessible limiting case which can be used to fix the integration constant. We have therefore adjusted the integration constant such that the integrated curve starts at the value where its left section ended. According to this integration, the maximal force during cleaving of the delocalized $\pi$ bond between PTCDA and $\operatorname{Ag}(111)$ is $0.5 \mathrm{nN}$.

Finally, we turn to the electrical conductance measurements through the PTCDA wire. Figure 3(d) shows the two-dimensional color-coded conductance histogram for the data set of Fig. 3(a), made with logarithmic bins. For $6 \AA<z<10 \AA$ the conductance behavior of the PTCDA wire has been reported and discussed before. ${ }^{2,12}$ Here we concentrate on the range $z>16 \AA$. At $z \approx 17 \AA$, we observe a sharp turning point at which the conduction through the molecular wire abruptly gives way to tunneling, the latter revealed by the characteristic exponential dependence of the conductance on the distance. This shows that precisely at the point of largest force across the wire junction a vacuum gap opens between the molecular wire and the surface. Through the last angstrom before the transition to tunneling, we observe a plateau in the conductance, which should correspond to the conductance of a PTCDA wire that is contacted by its carboxylic oxygens at one end to the tip and at the other end to the $\operatorname{Ag}(111)$ surface [Fig. 1(e)]. Interestingly, on this plateau the distribution of wire conductances exhibits two well-defined peak values, $G_{1}=(1.3 \pm 0.3) \times 10^{-5} G_{0}$ 
and $G_{2}=(0.6 \pm 0.1) \times 10^{-5} G_{0}$, where $G_{0}$ is the quantum of conductance $(12.9 \mathrm{k} \Omega)^{-1}$. This indicates that there exist two stable configurations of the molecular wire junction. Note that the two conductances vary by nearly a factor of 2 , that is, $\frac{G_{1}}{G_{2}} \approx$ 2.1. One may speculate that these configurations have one or two (carboxylic) oxygen atoms, respectively, in contact with the tip electrode.

The method used here for the characterization of a prototypical molecular wire provides independent force-based control over the conformation of the molecule in the junction. The large stiffness and the low oscillation amplitudes of the qPlus sensor make it a unique tool for single-molecule transport studies. The experimental strategy described here is directly applicable to the broad class of molecules that are composed of the nanometer-sized graphene ribbons or flakes and functionalized with carboxylic or other groups of sufficient reactivity as contacts to the tip. Since this type of molecular wire is of practical importance, ${ }^{11}$ their systematic study is highly desirable. Furthermore, the case of PTCDA analyzed here inspires optimism since it demonstrates that molecular wires can sustain stable electrical contacts between two macroscopic electrodes even if one of the leads is subjected to mechanical oscillations with amplitudes of about $10 \%$ of the total length of the wire. At the same time, the rather low conductance of the present wire should improve as the size of the conducting nanoribbon and/or the number of carboxylic clamps per contact increases.

We gratefully acknowledge fruitful discussions with M. Rohlfing (Universität Osnabrück) and C. Toher (TU Dresden), as well as the financial support from the Deutsche Forschungsgemeinschaft (DFG SPP 1243). R.T. acknowledges support by the Helmholtz-Gemeinschaft.
*Corresponding author: r.temirov@fz-juelich.de

${ }^{1}$ N. Néel, J. Kröger, L. Limot, T. Frederiksen, M. Brandbyge, and R. Berndt, Phys. Rev. Lett. 98, 065502 (2007).

${ }^{2}$ R. Temirov, A. Lassise, F. B. Anders, and F. S. Tautz, Nanotechnology 19, 065401 (2008).

${ }^{3}$ N. Néel, J. Kröger, L. Limot, and R. Berndt, Nano Lett. 8, 1291 (2008).

${ }^{4}$ L. Lafferentz, F. Ample, H. Yu, S. Hecht, C. Joachim, and L. Grill, Science 323, 1193 (2009).

${ }^{5}$ Y. F. Wang, J. Kröger, R. Berndt, H. Vazquez, M. Brandbyge, and M. Paulsson, Phys. Rev. Lett. 104, 176802 (2010).

${ }^{6}$ G. Schull, T. Frederiksen, A. Arnau, D. Sanchez-Portal, and R. Berndt, Nat. Nanotechnol. 6, 23 (2011).

${ }^{7}$ A. Nitzan and M. A. Ratner, Science 300, 1384 (2003).

${ }^{8}$ N. J. Tao, Nat. Nanotechnol. 1, 173 (2006).

${ }^{9}$ F. Chen, J. Hihath, Z. F. Huang, X. L. Li, and N. J. Tao, Annu. Rev. Phys. Chem. 58, 535 (2007).

${ }^{10}$ F. S. Tautz, Prog. Surf. Sci. 82, 479 (2007).

${ }^{11}$ F. Munoz-Rojas, J. Fernandez-Rossier, and J. J. Palacios, Phys. Rev. Lett. 102, 136810 (2009).

${ }^{12}$ C. Toher, R. Temirov, A. Greuling, F. Pump, M. Kaczmarski, M. Rohlfing, G. Cuniberti, and F. S. Tautz, Phys. Rev. B 83, 155402 (2011).

${ }^{13}$ F. J. Giessibl, Rev. Mod. Phys. 75, 949 (2003).

${ }^{14}$ See Supplemental Material at http://link.aps.org/supplemental/ 10.1103/PhysRevB.84.035435 for animation1.avi and animation2.avi.
${ }^{15}$ W. D. Cornell, P. Cieplak, C. I. Bayly, I. R. Gould, K. M. Merz, D. M. Ferguson, D. C. Spellmeyer, T. Fox, J. W. Caldwell, and P. A. Kollman, J. Am. Chem. Soc. 117, 5179 (1995).

${ }^{16}$ A. Hauschild, K. Karki, B. C. C. Cowie, M. Rohlfing, F. S. Tautz, and M. Sokolowski, Phys. Rev. Lett. 94, 036106 (2005).

${ }^{17}$ A. Hauschild, K. Karki, B. C. C. Cowie, M. Rohlfing, F. S. Tautz, and M. Sokolowski, Phys. Rev. Lett. 95, 209602 (2005).

${ }^{18}$ R. Rurali, N. Lorente, and P. Ordejon, Phys. Rev. Lett. 95, 209601 (2005).

${ }^{19}$ P. Fenter, F. Schreiber, L. Zhou, P. Eisenberger, and S. R. Forrest, Phys. Rev. B 56, 3046 (1997).

${ }^{20}$ J. Ikonomov, P. Bach, R. Merkel, and M. Sokolowski, Phys. Rev. B 81, 161412 (2010).

${ }^{21}$ A. Kraft, R. Temirov, S. K. M. Henze, S. Soubatch, M. Rohlfing, and F. S. Tautz, Phys. Rev. B 74, 041402(R) (2006).

${ }^{22}$ M. Ternes, C. P. Lutz, C. F. Hirjibehedin, F. J. Giessibl, and A. J. Heinrich, Science 319, 1066 (2008).

${ }^{23}$ M. Ternes, C. Gonzalez, C. P. Lutz, P. Hapala, F. J. Giessibl, P. Jelinek, and A. J. Heinrich, Phys. Rev. Lett. 106, 016802 (2011).

${ }^{24}$ The simulated $\Delta f(z)$ curve was obtained as $\Delta f=\frac{d^{2} V_{\text {total }}}{d z^{2}} \times \frac{f_{0}}{2 k_{0}}$. The resultant curve was smoothed to remove numerical noise.

${ }^{25}$ The determination of the absolute $z_{\text {tip }}$ scale in the experiment was done as reported in Ref. 12.

${ }^{26}$ A. Noy, Surf. Interface Anal. 38, 1429 (2006).

${ }^{27}$ M. Rief, F. Oesterhelt, B. Heymann, and H. E. Gaub, Science 275 1295 (1997).

${ }^{28}$ T. Hugel and M. Seitz, Macromol. Rapid Commun. 22, 989 (2001). 